\title{
IMPLEMENTACIÓN Y OPERACIÓN DEL HORNO DE INDUCCIÓN DE LA ESME
}

\section{IMPLEMENTATION AND OPERATION OF THE ESME INDUCTION FURNACE}

\author{
Zoilo Valdivia Infantas'; Carlos Gutiérrez Delgado'
}

\begin{abstract}
RESUMEN
El trabajo pretende suministrar la información técnica como especificaciones, planos, etc. necesarios para que OPI pueda elaborar las bases para la implementación de los dos hornos de inducción de la ESME, asimismo considera la construcción de las obras civiles de uno de ellos, de modo que sirva de referencia para las obras civiles del segundo. La implementación de ambos permitirá que los alumnos de la Escuela de Metalurgia realicen prácticas académicas en los cursos de Siderurgia y Fundición, así como para tener oportunidad de investigación de nuevas aleaciones en el campo de los aceros y el hierro fundido resolver problemas de materiales muy especializados que pudiera requerir la industria, sobre todo minera. Con el horno montado en su base, se podrá realizar la instalación eléctrica y puesta en marcha por parte de técnicos especialistas.
\end{abstract}

Palabras clave: Horno de inducción, fundición

\section{RESUMEN}

The project supplies the technical information such as specifications, drawings, etc. necessary for developing the implementation of the two induction furnace of the ESME, also the project considers the construction of the civilians for one of the two furnaces, so serve as a reference for the civil works of the second furnace, The implementation of the two furnaces will allow that the students of metallurgy can make academic practices in the courses of iron and steel, also to provide the opportunity to research new alloys in the field of steel and cast iron and the possibility of solving problems of very specialized materials that could require the industry especially mining. Mounted the ovens electric installation could be made and specialized technicians will start the project.

Keywords: Inducction furnace, foundry

\section{I.- INTRODUCCIÓN}

Consideramos importante poner operativo el Horno de Inducción de 150 kilos para producir aceros y hierro fundido. Se trata de un equipo necesario para la formación académica de los alumnos en las áreas de Siderurgia y, sobre todo, en la de Fundición. Existen más de 200 fundiciones en el país y permanentemente son una fuente de puestos de trabajo para muchos egresados, quienes, a corto plazo se incorporarán a la actividad profesional con una mejor preparación y con grandes posibilidades de éxito.

Este horno se encuentra encajonado desde hace tres años en el Taller de la ESME. Su implementación requiere de varias acciones, así, un estudio para su implementación, con planos de instalación detallados que permitan llevar a cabo la obra civil y las acciones complementarias que no fueron consideradas en su adquisición.

Para poner en operación el Horno de Inducción elaboramos el presente proyecto de Implementación y Operación del Horno de Inducción de la ESME. El cual servirá para que la OPI ejecute las obras civiles y adquiera los equipos complementarios para el montaje y ponga en marcha de los dos hornos de inducción de la ESME. 


\section{II.- MATERIAL Y MÉTODOS}

\section{.2.1. Materiales}

Para la obra civil de referencia de uno de los hornos se empleará arena y piedras del mismo suelo y cemento donado por los profesores y alumnos del cuarto año de Metalurgia.

\subsection{Metodología}

El desarrollo del proyecto requirió de las siguientes acciones:

- Elaboración los planos de instalación en forma más detallada.

- Construcción de la obra civil, de uno de los dos hornos que servirá como referencia para el segundo horno y para establecer con mayor precisión los requerimientos totales de la implementación de los dos hornos de inducción.

- Suministración de toda la información técnica a OPI para que proceda a cotizar y otorgar la buena pro para la implementación de los dos hornos de inducción.

- Montaje de uno de los hornos sobre su base.

- Alineamiento y prueba manualmente del sistema de basculamiento.

- Elaboración del manual de operación, de seguridad y de mantenimiento.

\section{III.- RESULTADOS}

- Se construyó la cimentación de uno de los hornos así como el pozo para el condensador.

- Se suministró toda la información técnica a OPI para que proceda a cotizar y otorgar la buena pro para la implementación de los dos hornos de inducción.

- Se montó uno de los dos hornos de inducción.

- Se procedió a alinear el horno montado así como probar su sistema de basculamiento.

-Se elaboró el manual de operación, mantenimiento y seguridad de los hornos de inducción.

\section{IV.- DISCUSIÓN DE RESULTADOS}

- Los planos generales del fabricante no son lo suficientemente precisos ni se adecuan a las necesidades específicas de los usuarios, es el caso de la realización de prácticas académicas de los alumnos de metalurgia, sobre todo en aspectos de sencillez, seguridad y cronograma de uso.

- La alternancia de uso de los dos hornos, de ningún modo pueden trabajar simultáneamente. Este hecho ha sido discutido con los técnicos especialistas, sobre todo, en el aspecto de una capacitación para realizar el cambio sin problemas.

- La operación del horno es sencilla, al alcance de los alumnos, pero requiere de una supervisión exigente por parte de los docentes.

- La instalación tiene un alto ciclo de vida, sólo que no debe descuidarse su mantenimiento es aspectos de limpieza y prevención de choques térmicos.

\section{V.- CONCLUSIONES}

- Se han elaborado los planos de detalle para la obra civil.

- Se ha elaborado el estudio técnico en el que se indica todos los requerimientos, tanto técnicos como de materiales y servicios para que puedan operar los dos hornos de inducción; información necesaria para proceder a las adquisiciones respectivas.

- Se ha demostrado la necesidad de instalar una nueva subestación con su transformador, para uso exclusivo del horno de inducción.

- Se ha suministrado información necesaria faltante para el cierre del Proyecto de Implementación de los Laboratorios de la ESME.

- Se ha realizado el montaje de uno de los hornos de inducción, que servirá como modelo para el montaje del segundo horno.

- Se ha elaborado los planos para la construcción de la plataforma de trabajo.

- Se ha elaborado el manual de operación, mantenimiento y seguridad del horno de Inducción.

- Se ha suministrado toda la información necesaria a OPI para que proceda a licitar la nueva subestación y los servicios técnicos especializados para las conexiones eléctricas de 
los hornos y la elaboración del sinterizado de los crisoles, así como el montaje y puesta en operación de los dos hornos de inducción, que servirán para la realización de las prácticas de los cursos de Siderurgia y Fundición en condiciones similares a las que le ofrecería la Industria.

- Los profesores tendrán la oportunidad de realizar investigación de nuevas aleaciones en el campo de los aceros y el hierro fundido.

- Se podrán elaborar algunas piezas fundidas que puedan resolver necesidades de repuestos para algunos equipos en la ESME y de la UNJBG.

- Se podrán resolver problemas de materiales muy especializados que pudiera requerir la industria, sobre todo minera.

\section{VI.- RECOMENDACIONES}

- Se deberán realizar las adquisiciones de los materiales para las prácticas de los alumnos con la debida anticipación, teniendo en cuenta cantidad mínima de compra, transporte, periodo de compra, etc.

- Los alumnos y profesores realizarán las operaciones con todos los implementos de seguridad y una capacitación rigurosa.

- Se deberá programar una limpieza periódica a toda la parte eléctrica, como subestación, cabina de control, contactos, etc.

- Las bombas deberán ser inspeccionadas antes de una operación que se realiza después de un mes de parada.

\section{REFERENCIAS BIBLIOGRÁFICAS}

- Astigarraga Urquizo Julio. Mexico 1994. Hornos industriales de inducción, Editorial MacGraw-Hill.

- China Electric (Shanghai) CO, LTD. 2009. Operating Manual for $0.15 \mathrm{t} \mathrm{mf}$, Induction Furnace.

- Shanghai Zhaoli Electrical Equipment manufacturing Co., Ltd. 2009. The Instruction for $\mathrm{KgpS}(\mathrm{F})$ Rapid Constant Power Middle Frecuency Power Supply.

\section{Correspondencia:}

Zoilo Valdivia Infantas

zoivaldivia@hotmail.com

Ciudad Universitaria fundo "Los Granados"

Av. Miraflores s/n Tacna - Perú

Carlos Gutierrez Delgado

cargdel@gmail.com

Ciudad Universitaria fundo "Los Granados"

Av. Miraflores s/n Tacna - Perú

\section{ANEXOS DE IMÁGENES REFERENTES ALTRABAJO DE INVESTIGACIÓN}

EN LOS GRÁFICOS A, B Y C SE MUESTRAN LOS PLANOS DE LA OBRA CIVIL

\section{A}

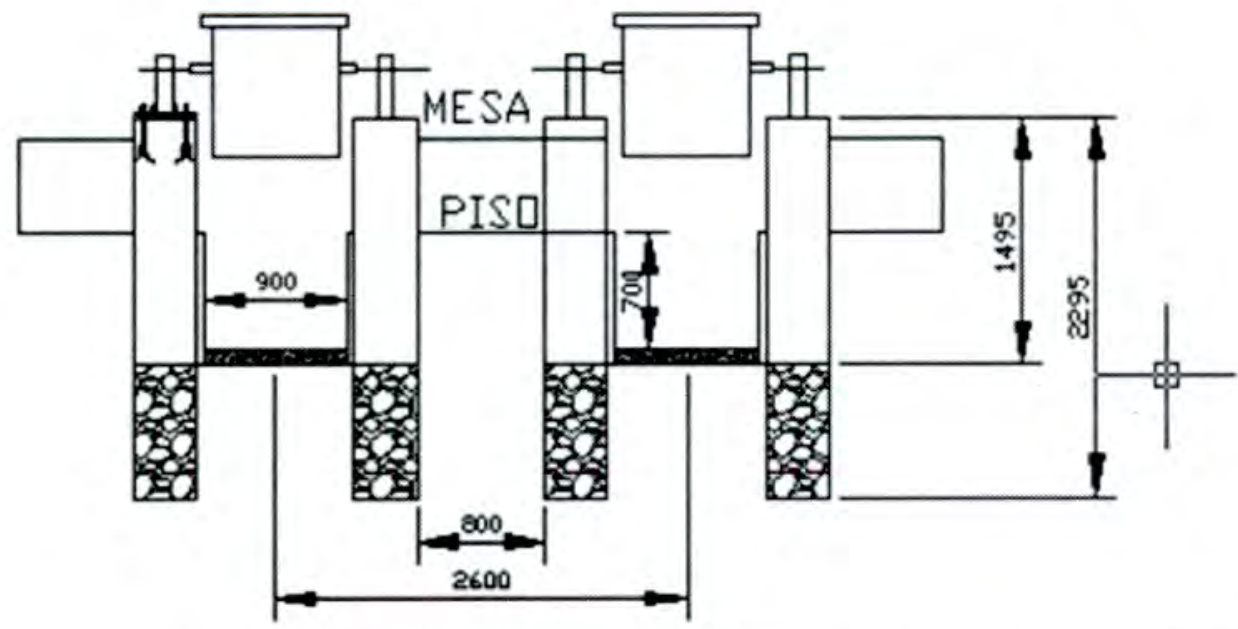


B

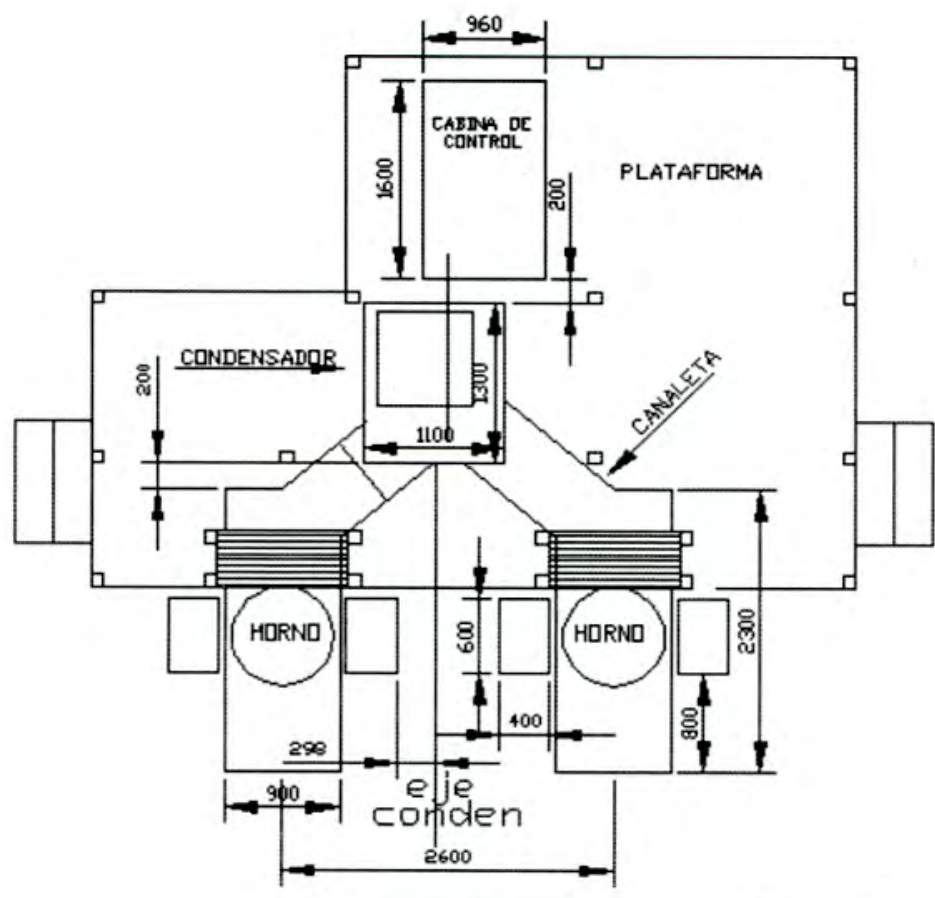

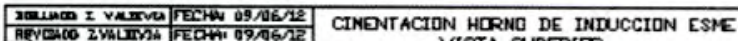

Squat La? VISTA SUPERTR

C

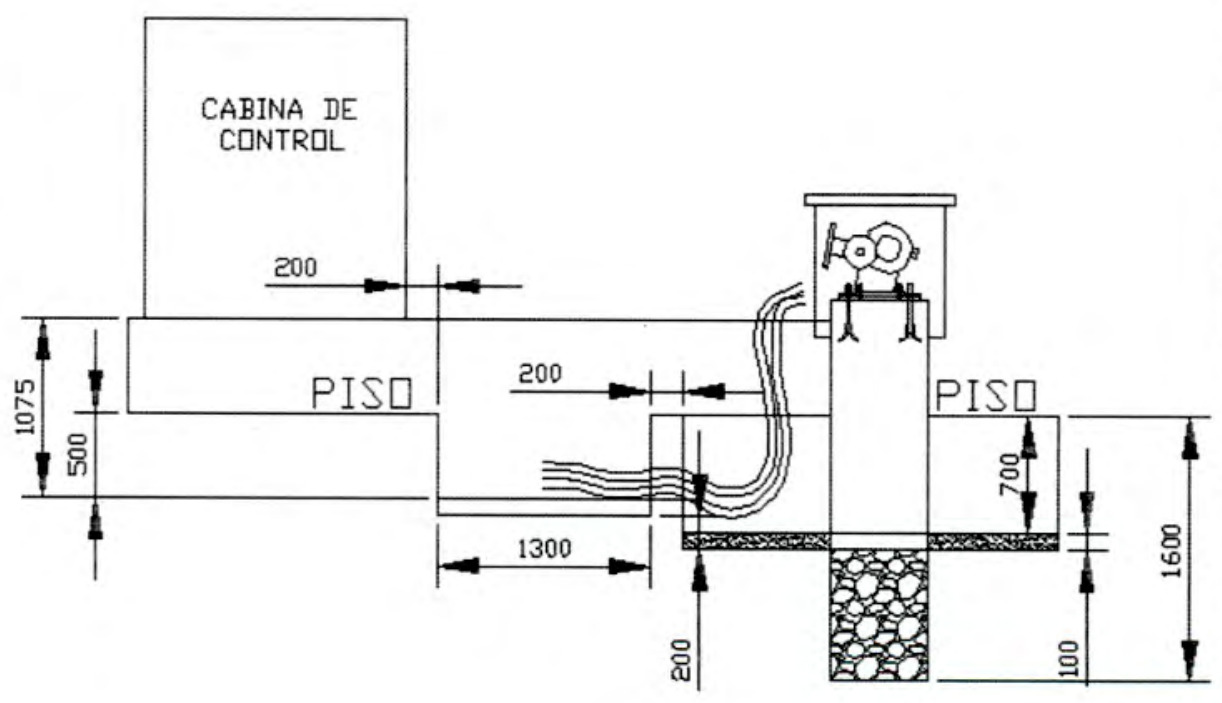


FOTOS DE LA CONSTRUCCIÓN DE LA CIMENTACIÓN DEL HORNO DE INDUCCIÓN

Encofrado de la obra civil

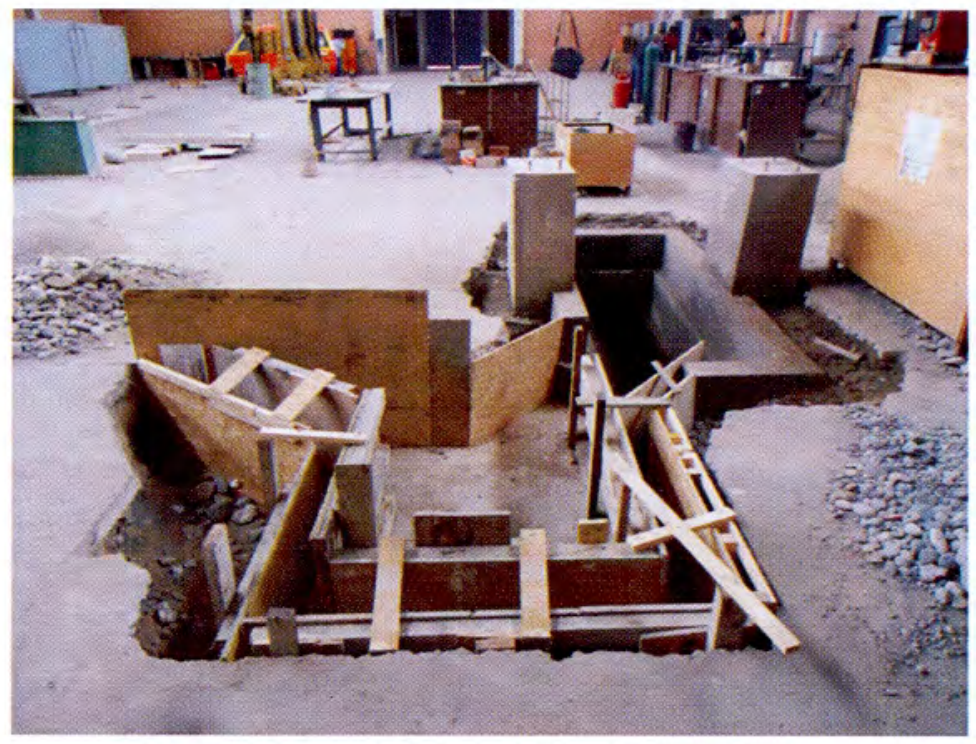

Vaciado de la obra civil.

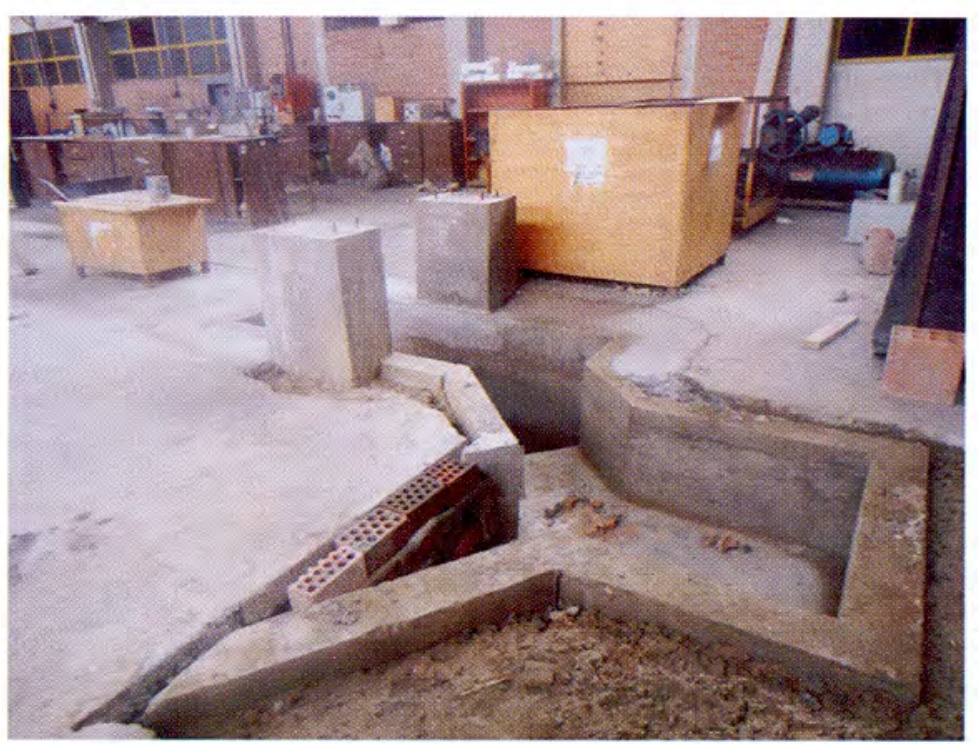

Vista del horno montado y condensador

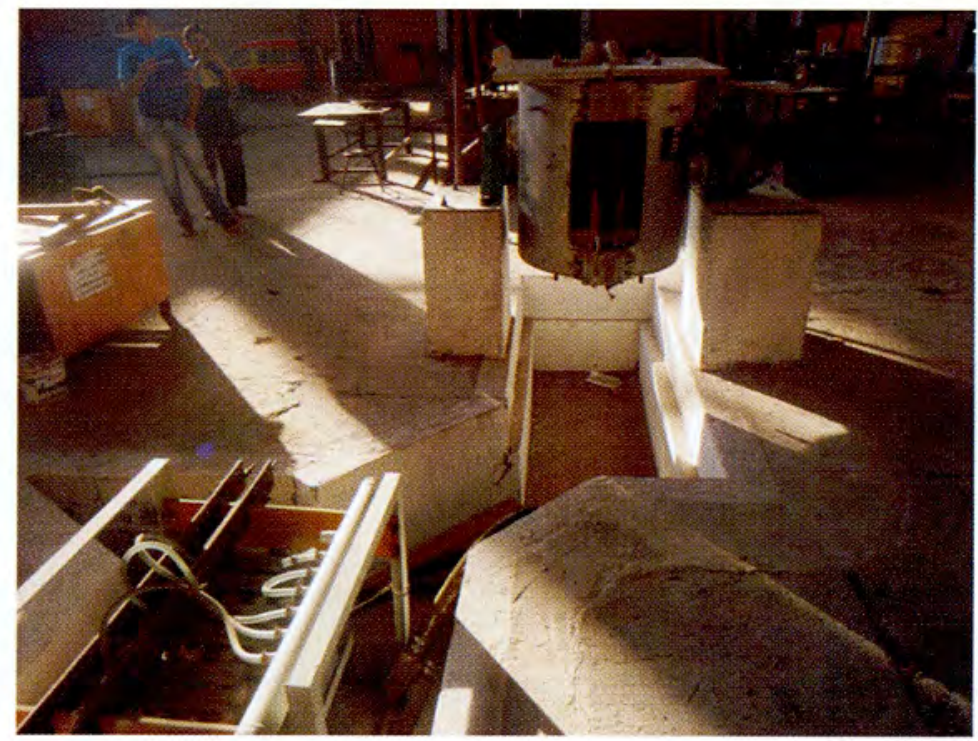

